\title{
CHARACTERISTIC POLYNOMIALS
}

\author{
HANS SCHNEIIEER
}

Introduction. Let $F$ be a field and let $V$ be a finite dimensional vector space over $F$ which is also a module over the ring $F[\mathbf{a}]$. Here a may lie in any extension ring of $F$. We do not assume, as yet, that $V$ is a faithful module, so that $\mathbf{a}$ need not be a linear transformation on $V$. It is known that by means of a decomposition of $V$ into cyclic $F[\mathbf{a}]$-modules we may obtain a definition of the characteristic polynomial of $\mathbf{a}$ on $V$ which does not involve determinants. In this note we shall give another non-determinantal definition of the characteristic polynomial. Instead of considering a single module $V$, we shall accordingly study the set of all finite dimensional $F[\mathbf{a}]$-modules and mappings of this set into monic polynomials with coefficients in $F$. Admittedly our procedure does not yield the theory of the elementary divisors of $\mathbf{a}$, but it has certain advantages. First, all questions of uniqueness are settled immediately by the Jordan-Hölder theorem. Secondly, it is possible to derive some classical results, usually proved using determinants, without excessive labour. To illustrate the use of our method we shall complete and generalise some results due to Goldhaber (2) and Osborne (5).

1. A principal ideal in a semigroup of mappings. Let $\mathfrak{B}$ be the set of all finite dimensional $F[\mathbf{a}]$-modules, and let $\mathfrak{M}$ be the multiplicative semigroup of (non-zero) monic polynomials with coefficients in $F$. Let $\mathfrak{I}$ be the set of mappings of $\mathfrak{B}$ into $\mathfrak{M}$. We shall assume throughout that if $\psi$ is a mapping of $\mathfrak{I}$, and if $V_{1}$ and $V_{2}$ are isomorphic modules of $\mathfrak{B}$, then $\psi\left(V_{1}, t\right)=\psi\left(V_{2}, t\right)$. The set $\mathfrak{I}$ becomes a semigroup if we define multiplication in the obvious way, viz. $\psi_{1} \psi_{2}(V, t)=\psi_{1}(V, t) \cdot \psi_{2}(V, t)$ for all $V$ in $\mathfrak{B}$. The subset $\mathfrak{S}$ of $\mathfrak{T}$ consisting of all mappings satisfying

$$
\psi(V, t)=\psi(V / Z, t) \cdot \psi(Z, t)
$$

for all $V \in \mathfrak{B}$ and all submodules $Z$ of $V$, is also a semigroup. Now let $\mu$ be any mapping of $\mathfrak{I}$ such that $\mu((0), t)=1$ and

$$
\mu(V, t) \text { divides } \mu(V / Z, t) \cdot \mu(Z, t)
$$

for all $V \in \mathfrak{B}$ and all submodules $Z$ of $V$. Let $\mathfrak{C}$ be the ideal in the semigroup $\mathfrak{S}$ consisting of all $\psi$ of $\mathfrak{S}$ divisible by $\mu$ in $\mathfrak{T}$ i.e. let $\mathfrak{S}=\mu \mathfrak{T} \cap \mathfrak{S}$.

Then an element $\psi$ of $\mathfrak{I}$ belongs to $\mathfrak{E}$ if and only if $\psi$ satisfies (1) and

$$
\mu(V, t) \text { divides } \psi(V, t)
$$

for all $V \in \mathfrak{B}$.

Received March 1, 1956. I should like to express my gratitude to I)r. I. T. Adamson and Dr. M. P. Drazin for discussing with me the results of this paper. 
Let $V=V_{0} \supset \ldots \supset V_{s}=(0)$ be a composition series for the $F[\mathbf{a}]$-module $V$. Then it is an immediate consequence of the Jordan-Hölder theorem that the polynomial

$$
\chi(V, t)=\prod_{i=1}^{s} \mu\left(V_{i-1} / V_{i}, t\right) \cdot \mu((0), t)
$$

depends only on the module $V$ and not on the composition series we use in the definition. (The last factor $\mu((0), t)=1$ has been put in to cover the case $V=(0)$, yielding $\chi((0), t)=\mu((0), t)=1$. In some of the arguments below it has been assumed that $V \neq(0)$, the case $V=(0)$ being trivial.)

This remark allows us to think of $\chi$ as a mapping of $\mathfrak{B}$ into $\mathfrak{M}$, i.e. as an element of the semigroup $\mathfrak{T}$. The semigroup $\mathfrak{S}$ is not a principal ideal semigroup. But we shall prove

Lemma 1. The mapping $\chi$ defined by (4) is the unique generator of the ideal (5) in $\subseteq$.

Proof. We shall first show that $\chi \in \subseteq$. If $Z$ is any submodule of $V$ then there is a composition series $V=V_{0}^{\prime} \supset \ldots \supset V_{s}^{\prime}=(0)$ in which $Z$ occurs, say $Z=V_{r}$. Thus it follows from our remarks about the JordanHölder theorem, and since $\left(V_{i-1}{ }^{\prime} / Z\right) /\left(V_{i}^{\prime} / Z\right)$ is isomorphic to $V_{i-1}{ }^{\prime} / V_{i}^{\prime}$ that

$$
\chi(V / Z, t) \chi(Z, t)=\prod_{i=1}^{r} \mu\left(V_{i-1}^{\prime} / V_{i}^{\prime}, t\right) \prod_{i=r+1}^{s} \mu\left(V_{i-1}^{\prime} / V_{i}^{\prime}, t\right)=\chi(V, t)
$$

whence $\chi \in \mathfrak{\Im}$.

It follows from (3) applied to the factor modules that

$$
\prod_{i=1}^{s} \mu\left(V_{i-1} / V_{i}, t\right)
$$

divides $\chi(V, t)$. Hence by $(2), \mu(V, t)$ divides $\chi(V, t)$. Thus $\mu$ divides $\chi$, and we deduce that $(\chi) \subseteq(5$.

To prove the reverse inclusion let us suppose that $\psi \in \mathfrak{C}$. Then for any $V \in \mathfrak{B}$, we have

$$
\psi(V, t)=\prod_{i=1}^{s} \psi\left(V_{i-1} / V_{i}, t\right)
$$

by (1). But, by (3), $\mu\left(V_{i-1} / V_{i}, t\right)$ divides $\psi\left(V_{i-1} / V_{i}, t\right)$, whence $\chi(V, t)$ divides $\psi(V, t)$. It follows that $\chi$ divides $\psi$ in $\mathfrak{I}$, say $\psi=\chi \psi^{*}$. To show that $\psi^{*}$ belongs to $\mathfrak{S}$ we observe that for all submodules $Z$ of $V$

$$
\begin{aligned}
& \chi(V, t) \psi^{*}(V, t)=\psi(V, t)=\psi(V / Z, t) \psi(Z, t) \\
& =\chi(V / Z, t) \quad \chi(Z, t) \psi^{*}(V / Z, t) \psi^{*}(Z, t)=\chi(V, t) \psi^{*}(V / Z, t) \psi^{*}(Z, t)
\end{aligned}
$$

whence $\psi^{*}$ satisfies $(1)$ since $\chi(V, t)$ is non-zero. We have now proved that $\psi \in(\chi)$, and this implies that $\mathfrak{S} \subseteq(\chi)$. It follows that $(\chi)=\mathfrak{S}$, and we conclude that $\chi$ is a generator of $\mathbb{S}$. 
To prove uniqueness, let us now suppose that $5=(\chi)=\left(\chi^{*}\right)$. Then $\chi^{*}=\chi \psi$ and also $\chi=\chi^{*} \psi^{*}$. We obtain that $\chi=\chi \psi \psi^{*}$, and it follows that $\psi \psi^{*}=\eta$, where $\eta(V, t)=1$, for all $V \in \mathfrak{B}$. The mapping $\eta$ is the identity of $\mathfrak{S}$ (and also, of course, of $\mathfrak{T}$ ), and since $\eta$ is the only inverse in $\mathfrak{T}$, it follows that $\psi=\eta$, whence $\chi=\chi^{*}$, and the lemma is proved.

COROLlary. If $\psi$ belongs to the ideal 5 , and $\psi(V, t)$ has the same degree as $\chi(V, t)$ for all $V$ in $\mathfrak{B}$, then $\chi=\psi$.

Proof. Clearly $\psi=\chi \psi^{*}$, where, for all $V$ in $\mathfrak{B}, \psi^{*}(V, t)$ is a monic polynomial of degree 0 , and the only such polynomial is 1 .

2. The definition of the characteristic polynomial. As is well known, the polynomials $p(t)$ in $F[t]$ for which $p(\mathbf{a}) V=0$ form an ideal in $F[t]$. By a slight extension of the usual terminology, we shall call the monic generator of this ideal the minimum polynomial of a on $V$, and denote it by $\mu_{a}(V, t)$. Thus $\mu_{a}$ is a mapping of $\mathfrak{B}$ into $\mathfrak{M}$ such that $\mu_{a}((0), t)=1$ and which satisfies (2) since

$$
\mu_{a}(Z, \mathbf{a}) \cdot \mu_{a}(V / Z, \mathbf{a}) V \subseteq \mu_{a}(Z, \mathbf{a}) Z=(0),
$$

when $Z$ is any submodule of $V$. It follows, therefore, that the ideal $\mathfrak{S}_{a}$, which consists of all $\psi \in \mathfrak{I}$ divisible by $\mu_{a}$, and which we shall call the characteristic ideal of $\mathbf{a}$ in $\Im$, is a principal ideal with a unique generator. Thus we may make the following definition:

Definition. Let $V \in \mathfrak{B}$. Then the characteristic polynomial of a on $V$ is $\chi_{a}(V, t)$, where $\chi_{a}$ is the unique generator of the characteristic ideal $\mathfrak{S}_{a}=\mu_{a} \mathfrak{I} \cap \mathfrak{S}$ of $\mathbf{a}$.

By virtue of Lemma 1, we obtain immediately

Theorem 1. Let $V \in \mathfrak{B}$, and let $V=V_{0} \supset \ldots \supset V_{s}=(0)$ be a composition series for $V$. Then the characteristic polynomial $\chi_{a}(V, t)$ of a on $V$ is

$$
\chi_{a}(V, \mathrm{t})=\prod_{i=1}^{s} \mu_{a}\left(V_{i-1} / V_{i}, t\right) \cdot \mu_{a}((0), t) .
$$

Corollary 1. For each $V$ in $\mathfrak{B}$, the degree of $\chi_{a}(V, t)$ equals the dimension of $V$.

Proof. In view of (5), we need only prove that the degree of $\mu_{a}(Z, t)$ equals the dimension of $Z$, when $Z$ is an irreducible $F[\mathbf{a}]$-module. This is well-known if $Z$ is faithful over $F[\mathbf{a}]$. If $Z$ is not faithful over $F[\mathbf{a}]$, then we must have $\mathbf{a} Z=0$. The dimension of $Z$ must equal 1 , and $\mu_{a}(Z, t)=t$.

Corollary 2. If $\psi$ belongs to the characteristic ideal of a, and if the degree of $\psi(V, t)$ equals the dimension of $V$ for each $V \in \mathfrak{B}$, then $\psi=\chi_{a}$.

This corollary follows from the previous one and from the corollary to Lemma 1. 
We shall now show that our definition of the characteristic polynomial leads to the usual one in the case of matrices. For any basis for $V$ we obtain a matrix of a on $V$ in the usual way. Since we have not assumed that $\mathbf{a}$ is a linear transformation on $V$, a non-zero a may have a zero matrix on $V$. The determinant of a matrix $A$ will be denoted by $|A|$.

Theorem 2. If $A$ is any matrix of a on $V$ and $I$ is the unit matrix, then (6)

$$
\chi_{a}(V, t)=|t I-A| \text {. }
$$

Proof. Any two basis for $V$ yield similar matrices of a on $V$. Hence the determinant $|t I-A|$ depends on $V$ only and not on the basis used to obtain $A$. Thus we may define a mapping of $\mathfrak{I}$ by setting $\psi(V, t)=|t I-A|$.

Let $Z$ be any submodule of $V$. We choose a basis for $Z$ and complete it to a basis for $V$. With respect to this basis the matrix of $\mathbf{a}$ on $V$ is

$$
B=\left[\begin{array}{ll}
B_{1} & B_{12} \\
0 & B_{2}
\end{array}\right],
$$

where $B_{1}$ is a matrix of a on $Z$, and $B_{2}$ is a matrix of $\mathbf{a}$ on $V / Z$.

Hence

$$
\psi(V, t)=|t I-B|=\left|t I-B_{1}\right|\left|t I-B_{2}\right|=\psi(Z, t) \psi(V / Z, t) ;
$$

and so $\psi$ belongs to $\subseteq$.

It is easily verified that $p(A)=0$ is equivalent to $p(\mathbf{a}) V=0$, whence the minimum polynomial of the matrix $A$ is just $\mu_{a}(V, t)$. Thus by the classical Cayley-Hamilton theorem applied to the determinant $|t I-A|$, the polynomial $\mu_{a}(V, t)$ divides $\psi(V, t)$. It follows that $\psi$ belongs to the characteristic ideal of $\mathbf{a}$. The degree of $\psi(V, t)$ is clearly equal to the dimension of $V$. We may now use Corollary 2 to Theorem 1 to conclude that $\chi_{a}(V, t)=\psi(V, t)=|t I-A|$.

We may remark that it is possible to obtain an extension of some of the above results to the case of $F[\mathbf{a}]$-modules of infinite dimension over $F$, in which case $V$ has no composition series and $\psi^{\prime}(V, t)=0$.

3. Characteristic polynomials with a common factor. In this section we shall again assume that the $F[$ a]-module $V$ is finite dimensional. If $Z$ is a submodule of $V$, then $\mu_{a}(V, t)$ is clearly divisible by both $\mu_{a}(V / Z, t)$ and $\mu_{a}(Z, t)$. But the product $\mu_{a}(V / Z, t) \cdot \mu_{a}(Z, t)$ is divisible by $\mu_{a}(V, t)$. It follows by $(5)$ that $\chi_{a}(V, t)$ divides a power of $\mu_{a}(V, t)$. Thus we have proved from our definitions the very well-known result that every irreducible factor of $\chi_{a}(V, t)$ is also a factor of $\mu_{a}(V, t)$. This enables us to prove the next lemma.

LEMMa 2. There exists a composition series for $V$ with the factor modules appearing in any order.

Proof. Let $p(t)$ be any irreducible factor of $\chi_{a}(V, t)$ and therefore also of $\mu_{a}(V, t)$. Let $q(t)=\mu_{a}(V, t) / p(t)$, and let $Z$ be the submodule of $V$ consisting of all $v \in V$ for which $q(\mathbf{a}) v=0$. Then $p(\mathbf{a}) V \subseteq Z$, and so $\mu_{a}(V / Z, t)$ divides 
$p(t)$. But $Z$ is a proper submodule of $V$, whence $\mu_{a}(V / Z, t) \neq 1$. We deduce that the minimum polynomial of a on $V / Z$ and any of its irreducible submodules is $p(t)$. Since we may start a composition series for $V$ with a composition series for $V / Z$, the lemma follows.

Lemma 3. Let $Z$ be a submodule of the $F[\mathbf{a}]$-module $V$, and let $Y$ be a submodule of the $F[\mathbf{a}]$-module $W$. Let $\lambda_{1}$ be a vector space isomorphism of $Y$ onto $Z$. If $V / Z$ and $W / Y$ are irreducible submodules for which $\mu_{a}(V / Z, t)=\mu_{a}(W / Y, t)$, then there exists an extension of $\lambda_{1}$ to a vector space isomorphism $\lambda$ of $W$ onto $V$ such that $\mathbf{a} w^{\lambda}-(\mathbf{b} w)^{\lambda} \in Z$, for all $w \in W$.

Proof. The conditions on $V / Z$ and $W / Y$ imply that there is a vector space isomorphism $\kappa$ of $W / Y$ onto $V / Z$ such that $\mathbf{a}(w+Y)^{\kappa}=(\mathbf{b}(w+Y))^{\kappa}$ for all $w \in W$. Let $Z^{\prime}$ be a subspace of $V$ complementary to $Z$, and let $Y^{\prime}$ be a subspace of $W$ complementary to $Y$. We shall now define a mapping of $W$ into $V$ as follows.

We let $\lambda$ coincide with $\lambda_{1}$ on $Y$. If $w \in Y^{\prime}$ we let $v=w^{\lambda}$ be the unique element of $Z^{\prime}$ for which $v+Z=(w+Y)^{\kappa}$. We then extend $\lambda$ linearly from $Y \cup Y^{\prime}$ to $W=Y+Y^{\prime}$. Then $\lambda$ is an isomorphism onto $V$, and the Lemma follows since, for all $w \in Y^{\prime}$, we have

$\mathbf{a} w^{\lambda}+Z=\mathbf{a}\left(w^{\lambda}+Z\right)=\mathbf{a}(w+Y)^{\kappa}=(\mathbf{b}(w+Y))^{\kappa}=(\mathbf{b} w+Y)^{\kappa}$

$$
=(\mathbf{b} w)^{\lambda}+Z \text {. }
$$

Let $\Omega(V)$ be the algebra over $F$ of linear transformations on the finite dimensional vector space $V$. If $W$ is also a vector space over $F$, and $\lambda$ is a vector space isomorphism of $W$ onto $V$, then $\lambda$ induces an isomorphosm $\rho$ of $\mathfrak{R}(W)$ onto $\mathfrak{R}(V)$, which may be defined by $\mathbf{b}^{\rho} w^{\lambda}=(\mathbf{b} w)^{\lambda}$, for all $w \in W$. Conversely, let $\rho$ be an isomorphism of $\mathbb{R}(W)$ onto $\mathcal{R}(V)$. If we consider $F$ as a subalgebra of both $\mathfrak{R}(W)$ and $\mathscr{R}(V)$ in the normal way, then elements of $F$ are left fixed by $\rho$. Hence it can be shown that $\rho$ is induced by an isomorphism $\lambda$ of $W$ onto $V(\mathbf{4}$, p. 237). It is this result which almost immediately yields the following lemma.

LEMma 4. Let $\rho$ be an isomorphism of the algebra of linear transformations $\mathcal{R}(W)$ onto $\mathfrak{R}(V)$. If $\mathbf{b} \in \mathcal{R}\left(W^{*}\right)$ and $\mathbf{c}=\mathbf{b}^{\rho}$, then $\chi_{b}(W, t)=\chi_{c}(V, t)$.

Proof. Let $\lambda$ be the isomorphism of the vector space $W$ onto $V$ which induces $\rho$. Then any composition series for the $F[\mathbf{b}]$-module $W$ is mapped by $\lambda$ into a composition series for the $F[\mathbf{c}]$-module $V$. It is easy to see that the minimum polynomial of $\mathbf{b}$ on a factor module of the first series equals the minimum polynomial of $\mathbf{c}$ on the corresponding factor module of the second series. The lemma now follows from Theorem 1.

This lemma is rather less trivial than may appear at first sight. For, if $\rho$ is an isomorphism merely of the subalgebra $F[\mathbf{b}]$ of $\mathfrak{R}(W)$ into $\&(V)$, and $\mathbf{c}=\mathbf{b}^{\rho}$, then we can only conclude that $\mu_{b}(W, t)=\mu_{c}(V, t)$. 
Goldhaber (2) and Goldhaber and Whaples (3) have proved that if $A$ and $B$ are square matrices with coefficients in $F$ such that there exists a non-singular matrix $P$ for which $N=A-P B P^{-1}$ lies in the radical of $F\left[A, P B P^{-1}\right]$ then $|t I-A|=|t I-B|$. This result is generally known as Goldhaber's lemma. By means of the theory of canonical matrices and under the assumption that $F$ is an infinite perfect field Osborne (5)!has proved this lemma together with its converse. From now on we shall assume that $\mathbf{a}$ and $\mathbf{b}$ are linear transformations on the finite dimensional vector spaces $V$ and $W$ respectively, and we shall prove a theorem equivalent to Osborne's without any restriction on the field $F$.

TheOREM 3. Let $\mathbf{a}$ and $\mathbf{b}$ be linear transformations on the finite dimensional vector spaces $V$ and $W$ respectively. Then the characteristic polynomial of a on $V$ equals the characteristic polynomial of $\mathbf{b}$ on $W$ if and only if there is an isomorphism $\rho$ of $\mathfrak{R}(W)$ onto $\mathfrak{L}(V)$ such that $\mathbf{n}=\mathbf{a}-\mathbf{b}^{\rho}$ lies in the radical of $F\left[\mathbf{a}, \mathbf{b}^{\rho}\right]$.

Proof. If $\rho$ is an isomorphism of $\mathfrak{R}(W)$ onto $\mathfrak{R}(V)$, and $\mathbf{c}=\mathbf{b}^{\rho}$, then by Lemma 4 we need only prove that $\chi_{a}(V, t)=\chi_{c}(V, t)$ when $\mathbf{n}=\mathbf{a}-\mathbf{c}$ lies in the radical of

$$
F[\mathbf{a}, \mathbf{c}]=F[\mathbf{a}, \mathbf{n}]=F[\mathbf{c}, \mathbf{n}] .
$$

Let $Z$ be an irreducible $F[\mathbf{a}, \mathbf{c}]$-module. Then $\mathbf{n} Z=(0)$, whence $Z$ is irreducible also over $F[\mathbf{a}]$ and $F[\mathbf{c}]$. Further, $\mu_{a}(Z, t)=\mu_{c}(Z, t)$, since for a polynomial $p(t)$ in $F[t]$ the equality $p(\mathbf{c}) Z=0$ implies $\left(p(\mathbf{a})+\mathbf{n}^{\prime}\right) Z=0$, where $\mathbf{n}^{\prime}$ belongs to the radical of $F[\mathbf{a}, \mathbf{c}]$, whence $p(\mathbf{a}) Z=0$; and conversely. Let

$$
V=V_{0} \supset \ldots \supset V_{s}=(0)
$$

be a composition series for the $F[\mathbf{a}, \mathbf{c}]$-module $V$. It follows from the remarks we have just made that this series is also a composition series for $V$ as an $F[\mathbf{a}]$ and $F[\mathbf{c}]$-module, and that

$$
\mu_{a}\left(V_{i-1} / V_{i}, t\right)=\mu_{c}\left(V_{i-1} / V_{i}, t\right), \quad i=1, \ldots, s .
$$

Hence by Theorem $1, \chi_{a}(V, t)=\chi_{c}(V, t)$.

Now let us suppose that $\chi_{a}(V, t)=\chi_{b}(W, t)$, and let $V=V_{0} \supset \ldots \supset V_{s}$ $=(0)$ be a composition series for the $F[\mathbf{a}]$-module $V$. We deduce from Lemma 2 that there is a composition series

$$
W=W_{0} \supset \ldots \supset W_{s}=(0)
$$

for the $F[\mathbf{b}]$-module $W$ such that $\mu_{b}\left(W_{i-1} / W_{i}, t\right)=\mu_{a}\left(V_{i-1} / V_{i}, t\right)$, for $i=1$, $\ldots, s$. Then using Lemma 3 we may prove by induction that there is a vector space isomorphism $\lambda$ of $W$ onto $V$ which takes $W_{i}$ onto $V_{i}$ such that aw $(\mathbf{b} w)^{\lambda} \in V_{i}$, whenever $w \in W_{i-1}(i=1, \ldots, s)$. Let $\rho$ be the isomorphism of $\mathfrak{R}(W)$ onto $\mathfrak{R}(V)$ associated with $\lambda$. If $v=w^{\lambda} \in V_{i-1}$, and $\mathbf{n}=\mathbf{a}-\mathbf{b}^{\rho}$, then

$$
\mathbf{n} v=\mathbf{a} w^{\lambda}-\mathbf{b}^{\rho} w^{\lambda}=\mathbf{a} w^{\lambda}-(\mathbf{b} w)^{\lambda} \in V_{i} .
$$


Thus for any polynomial $p(t)$ in $F[t]$ we have $(p(\mathbf{a}) \mathbf{n})^{s} V=(0)$. Since $V$ is faithful over $F\left[\mathbf{a}, \mathbf{b}^{\rho}\right]$, we conclude that $\mathbf{n}$ lies in the radical of $F\left[\mathbf{a}, \mathbf{b}^{\rho}\right]$.

Lemma 4 allows us to state Theorem 3 symmetrically: The characteristic polynomials $\chi_{a}(V, t)$ and $\chi_{b}(W, t)$ are equal if and only if there exist isomorphisms $\sigma$ and $\tau$ of $\mathfrak{R}(V)$ and $\mathfrak{R}(W)$ respectively onto an algebra of linear transformations $\mathfrak{Q}(X)$ such that $\mathbf{n}=\mathbf{a}^{\sigma}-\mathbf{b}^{\tau}$ lies in the radical of $F\left[\mathbf{a}^{\sigma}, \mathbf{b}^{\boldsymbol{\tau}}\right]$.

Let $\Im(Z, V)$ be the subalgebra of $\mathcal{Q}(V)$ consisting of all linear transformations $\mathbf{d} \in \Omega(V)$ for which $\mathbf{d} Z \subseteq Z$. We note that $\mathbf{d} \in \Im(Z, V)$ if and only if $Z$ is an $F[\mathbf{d}]$-module. The natural homomorphism $\mathbf{d} \rightarrow \mathbf{d}^{\prime}$ of $\mathfrak{I}(Z, V)$ onto $\mathcal{Q}(Z)$ is defined by $\mathbf{d}^{\prime} v=\mathbf{d} v$, for all $v \in Z$. Its kernel $K_{1}$ consists of all $\mathbf{d} \in$ $\mathfrak{Y}(Z, V)$ such that $\mathbf{d} Z=(0)$. Clearly $\chi_{a^{\prime}}(Z, t)=\chi_{a}(Z, t)$. There is also a natural homomorphism of $\Im(Z, V)$ onto $\left\{(V / Z)\right.$, defined by $\mathbf{d} \rightarrow \mathbf{d}^{\prime \prime}$ where $\mathbf{d}^{\prime \prime}(v+Z)=\mathbf{d} v+Z$ for all $v \in V$. Its kernel $K_{2}$ consists of all $\mathbf{d} \in 3(Z, V)$ such that $\mathbf{d} V \subseteq Z$. Again $\chi_{a^{\prime \prime}}(V / Z, t)=\chi_{a}(V / Z, t)$.

Lemma 5. Let $X$ be a vector space of dimension $r$ over $F$, and let $p(t)$ be a monic polynomial of degree $r$ in $F[t]$. Then $p(t)$ divides $\chi_{a}(V, t)$ if and only if there exists an $F[\mathbf{a}]$-submodule $Z$ contained in $V$ and a homomorphism $\sigma$ of $\Im(Z, V)$ onto $\mathbb{R}(X)$ such that $\chi_{c}(X, t)=p(t)$, where $\mathbf{c}=\mathbf{a}^{\sigma}$.

Proof. Let $p(t)$ be a factor of $\chi_{a}(V, t)$. By Lemma 2 there exists an $F[a]$ module $Z$ contained in $V$ for which $\chi_{a}(Z, t)=p(t)$, and by Corollary 1 to Theorem 1 the dimension on $Z$ is $r$. Thus we may define the homomorphism $\sigma$ of $\mathfrak{Y}(Z, V)$ onto $\mathfrak{R}(X)$ to be the composed map of the natural homomorphism $\mathbf{d} \rightarrow \mathbf{d}^{\prime}$ of $\mathfrak{\Im}(Z, V)$ onto $\mathbb{Q}(Z)$, and any isomorphism of $\mathcal{Q}(Z)$ onto $\&(X)$. Then putting $\mathbf{c}=\mathbf{a}^{\sigma}$, we have

$$
\chi_{c}(X, t)=\chi_{a^{\prime}}(Z, t)=\chi_{a}(Z, t)=p(t),
$$

by virtue of Lemma 4 .

Conversely, let $\sigma$ be a homomorphism of $\mathfrak{\Im}(Z, V)$ onto $\mathfrak{\imath}(X)$ for which $\chi_{c}(X, t)=p(t)$. Using the simplicity of $\&(X)$ it may be shown that the kernel $K$ of $\sigma$ is $\Im(Z, V), K_{1}$ or $K_{2}$. If $K=\Im(Z, V)$, then $\mathfrak{Q}(X)=(0)$, whence $X=(0)$ and $p(t)=1$. If $K=K_{1}$, then $\mathfrak{R}(Z)$ is isomorphic to $\mathfrak{R}(X)$ under an isomorphism which takes $\mathbf{a}^{\prime}$ onto $\mathbf{c}=\mathbf{a}^{\sigma}$. In this case we deduce that $p(t)=\chi_{a^{\prime}}(Z, t)=\chi_{a}(Z, t)$, and $\chi_{a}(Z, t)$ divides $\chi_{a}(V, t)$. If $K=K_{2}$, then $\mathfrak{Q}(V / Z)$ is isomorphic to $\mathbb{Q}(X)$ under an isomorphism taking $\mathbf{a}^{\prime \prime}$ onto $\mathbf{c}$, and so

$$
p(t)=\chi_{a^{\prime \prime}}(V / Z, t)=\chi_{a}(V / Z, t),
$$

which again divides $\chi_{a}(V, t)$.

By combining the symmetric form of Theorem 3 and Ieemma 5 we immediately obtain a generalisation of Theorem 3 . 
TheOREM 4. Let $\mathbf{a}$ and $\mathbf{b}$ be linear transformations on the vector spaces $V$ and $W$ respectively. Let $X$ be a vector space of dimension $r$ over $F$. Then the characteristic polynomials $\chi_{a}(V, t)$ and $\chi_{b}(W, t)$ have a common factor of degree $r$ if and only if there exist an $F[\mathbf{a}]$-module $Z$ contained in $V$ and a homomorphism $\sigma$ of $\Im(Z, V)$ onto $\mathfrak{R}(X)$, an $F[\mathbf{b}]$-module $Y$ contained in $W$ and a homomorphism $\tau$ of $\mathfrak{S}(Y, W)$ onto $\mathfrak{R}(X)$, such that $\mathbf{n}=\mathbf{a}^{\sigma}-\mathbf{b}^{\tau}$ lies in the radical of $F\left[\mathbf{a}^{\sigma}, \mathbf{b}^{\tau}\right]$.

Finally, we claim that some of the results of Goddard and Schneider (1) and other results on characteristic polynomials may be derived from Theorem 4 .

\section{REFERENCES}

1. L. S. Goddard and H. Schneider, Matrices with a non-zero commutator, Proc. Cambridge Phil. Soc. 51 (1955), 551-553.

2. J. K. Goldhaber, The homomorphic mapping of certain matric algebras onto rings of diagonal matrices, Can. J. Math. 4 (1952), 31-42.

3. J. K. Goldhaber and G. Whaples, On some matrix theorems of Frobenius and McCoy, Can. J. Math. 5 (1953), 332-335.

4. N. Jacobson, Lectures on abstract algebra, vol. 2 (New York, 1953).

5. E. E. Osborne, On matrices having the same characteristic equation, Pacific J. Math. 2 (1952), 227-230.

Queen's University, Belfast and

Washington State College

Pullman, Wash. 